\title{
The emergence of the novel H1N1 virus: implications for global mental health
}

The emergence of the novel swine-origin influenza A (H1N1) virus in humans has aroused great concern among medical professionals about the possible evolution of a full-blown influenza pandemic, one on the scale of the "Spanish" influenza pandemic of 1918-19 (Belshe, 2009). It has been speculated that the return of a pandemic virus equivalent in pathogenicity to the virus of 1918 would likely kill more than 100 million people worldwide, including a large number of economically active young people (Taubenberger and Morens, 2006). Health administrations worldwide have stepped up reporting and surveillance of the deaths and illnesses associated with $\mathrm{H} 1 \mathrm{~N} 1$, and most countries have national strategies to fight the outbreak, though skeptics doubt how such plans could be operationalized, especially in developing countries (Coker, 2009). As of 6 July 2009 , the cumulative total of $\mathrm{H} 1 \mathrm{~N} 1$ cases exceeds 90,000 in over 100 countries, with over 400 deaths directly related to the infection (World Health Organization, 2009a). Optimists might believe this pandemic is not going to match the scale of the historical 1918 pandemic given the relatively low fatality rate observed thus far. However, the World Health Organization has cautioned that we have just entered Phase 6 of the pandemic -i.e. we are in the early days of the 2009 flu pandemic (Chan, 2009). The course of the pandemic is thus unpredictable at this stage but it is evident that international multilateral plans and agreements have enabled much greater coordination of communication and action than ever before. The guidance behind these multilateral international actions, rooted in the World Health Organization's International Health Regulations (IHR) 2005, only came into being five years ago in response to the threat of emerging infectious diseases and particularly by the events related to the emergence of Severe Acute Respiratory Syndrome (SARS) (Katz, 2009). The morbidity and mortality directly resulting from this novel influenza A H1N1 outbreak are in the center of the world media's spotlight, but the potential impact of the pandemic on global mental health has not yet received the attention it deserves.

First published online 14 September 2009
In 2003 the emergence of a novel virus causing SARS afflicted 8098 individuals and took 774 lives across 29 countries concentrated mainly in Asia (World Health Organization, 2003). The city of Hong Kong (Special Administrative Region of China), home to 6.8 million people, bore the brunt of the disease burden and case fatality: 1755 cases and 302 deaths occurred from 15 February to 31 May 2003 (Leung et al., 2004). The SARS outbreak overloaded the health care system in Hong Kong and depressed the economy further. Medical resources were channeled into combating SARS at the expense of routine non-emergency health care services. Mental health service provision at all levels (emergency service, inpatient and outpatient units, community outreach service, rehabilitative service, relapse prevention service, early intervention service) were considered low priority on the public health agenda during the SARS outbreak. While resources were diverted away from the mental health service sector, new demands were surging as the SARS epidemic caused mass fear, breakdown of social networks, and limited access to the health care system, which in turn may have triggered a cascade of social, physical and mental health problems and probably increased the suicide risk. The compromised mental health system failed to meet the excess psychiatric morbidity and mortality engendered during the outbreak.

These views are based on the empirical evidence from previous observational studies that used Poisson regression to analyze mortality data in Hong Kong (Chan et al., 2006; Cheung et al., 2008). Against the background of a downward trend in elderly suicide rates from 1986 through 2002 (steady decline from 40 per 100,000 to a trough level of around 29-31 per 100,000 in 1999 to 2002), an upturn in the suicide rate was observed in 2003 to over 40 per 100,000 . Poisson regression analysis showed that the rise was statistically significant (Chan et al., 2006; Cheung et al., 2008). Also, the number of suicide deaths in older adults (aged 65 and over) peaked in April 2003 when the highest number of incident SARS cases was documented (Chan et al., 2006; Cheung et al., 2008). Poisson regression analysis suggested that the observation of a peak suicide rate in April 2003 was in excess of a normal seasonal variation (Chan et al., 2006; 
Cheung et al., 2008). During the SARS outbreak in Hong Kong, the 2003 suicide rate in younger people (under 65 years) also increased but the difference from the previous year did not reach statistical significance (Chan et al., 2006). Hence, in the current $\mathrm{H} 1 \mathrm{~N} 1$ outbreak, older adults could prove to be the most vulnerable group. This observation is particularly alarming as the global population is "graying". It is estimated that the population in the over-60 age group will reach 1 billion, with $70 \%$ residing in developing countries, by 2020 .

The Hong Kong SARS story shows that given a relatively well-developed mental health system, lack of preparedness for mental health challenges arising from an outbreak could translate into immediate public health issues, adding to the fatality load of the whole community. The features common to both the SARS epidemic and potential flu pandemics are the potential for high fatalities, the high transmissibility and the susceptibility to the disease in the healthy population. Although the 2003 SARS experience in Hong Kong did not show any statistical association between excess suicide deaths in younger age groups (under 65 years) and the outbreak, one cannot assume that the psychological vulnerability of this group will not be tested amidst this evolving pandemic. When the global economy is struggling to find a way out of the worst financial crisis since the 1930s, the pandemic might well impact on the mental health of the economically active population through economic depression. Older adults, being economically, emotionally or physically dependent on their economically active family members, are likely to become secondary victims as a result. People across different age groups are thus vulnerable to the pandemic's potential impact on global mental health, albeit at varying degrees, with older adults being in the highest risk group.

According to the World Health Organization, suicide and mental illness are major public health issues, with both suicide and depression contributing significantly to the global loss of disability-adjusted life years (World Health Organization, 2000; 2009b). Medical professionals and health administrations may not be adequately prepared to meet the excess demands for mental health care when health resources are strained under critical situations like the pandemic or other novel outbreaks. On the other hand, massive outbreaks also pose specific challenges to existing mental health service. This is especially critical in developing countries where mental health resources are lacking or rudimentary. Mental health systems, if they exist at all, remain predominantly institutionally based in developing countries with little support for community-based psychiatric care. Psychiatric institutions in developing countries are thus prone to overcrowding, making them far from ideal when it comes to infection control. While old age psychiatry has been a rapidly growing field in developed countries over recent decades, it remains a low priority in the developing world which hosts the majority of the world's elderly population (Camus et al., 2003). Older people with dementia, especially those in developing countries, are thus the highest risk group during outbreaks of disease as they are the least cooperative, on account of their illness, in complying with infection control measures. They are thus susceptible to contracting the infection and spreading the disease to healthcare workers and other caregivers. Outbreaks in psychiatric institutions will disrupt standard mental health care provision and may result in the premature discharge of psychiatric patients to communities, which in turn may trigger a cascade of adverse clinical outcomes such as suicide.

Irrespective of the level of socioeconomic development of different nations, mental health preparedness for unusual events such as a massive outbreak or pandemic should be developed now and incorporated into action plans. We appreciate that compromise within the mental health service is inevitable in these situations when resources are strained. Yet it is no excuse for not rising to meet the challenge of the excess and specific mental health needs of the community. Mental health professionals are in the best position to take the lead in advising their regional health administrations as well as negotiating with other stakeholders to achieve coordination of effort. Detailed and practical contingency plans should exist at macro- and micro-levels in each operational unit of the mental health system, with designated personnel being responsible within each relevant sector. It is clear that there is no "one-size-fitsall" solution for different societies. Any feasible and optimal contingency plan should be based on systematic evaluation of what is already available in the form of mental health resources across primary, secondary and tertiary levels within a society's unique sociocultural settings (this also includes review of existing collaborative networks among the health sector, welfare sector, other non-governmental organizations and community gate-keepers), and the specific mental health needs of their communities (e.g. updates on the epidemiology of severe mental illness and common mental disorders, or the local perceptions of psychological distress related to massive outbreaks). The resource constraints during a massive outbreak should also be taken into account when planning service logistics and the human resources involved. The International Psychogeriatric Association and other international academic organizations may 
serve as platforms for the exchange and transfer of ideas and skills from developed countries to less developed parts of the world.

The course of the current pandemic will be unfolding in the coming months. Even if the current pandemic is contained or mitigated, there is still an urgent need for health administrations worldwide to establish transdisciplinary mental health strategies with their local mental health professionals in order to respond to different levels of outbreak both now and in the future.

\section{SANDRA S. CHAN, LINDA C. W. LAM AND HELEN F. K. CHIU \\ Department of Psychiatry, The Chinese University of Hong Kong, Hong Kong SAR, China \\ Email: schan@cuhk.edu.hk}

\section{References}

Belshe, R. B. (2009). Implications of the emergence of a novel H1 influenza virus. New England Fournal of Medicine, 360, 2667-2668. doi:10.1056/NEJMe0903995.

Camus, V. et al. (2003). Teaching and training in old age psychiatry: a general survey of the World Psychiatric Association member societies. International fournal of Geriatric Psychiatry, 18, 694-699. doi:10.1002/gps.900.

Chan, M. (2009). Influenza A (H1N1): lessons learned and preparedness. Keynote speech by Director-General of the WHO, Cancun, Quintana Roo, Mexico, 2 July 2009. Available at: http://www.who.int/dg/speeches/2009/ influenza_h1n1_lessons_20090702/en/index.html.

Chan, S. S. M., Chiu, H. F. K., Lam, L. C. W., Leung, V. P. Y. and Conwell, Y. (2006). Elderly suicide and the
2003 SARS epidemic in Hong Kong. International fournal of Geriatric Psychiatry, 21, 113-118. doi:10.1002/gps. 1432.

Cheung, Y. T., Chau, P. H. and Yip, P. S. F. (2008). A revisit on older adults' suicide and SARS epidemic in Hong Kong. International fournal of Geriatric Psychiatry, 23, 1231-1238. doi:10.1002/gps.2056.

Coker, R. (2009). Swine flu: fragile health systems will make surveillance and mitigation a challenge. BMF, 338, b1791. doi:10.1136/bmj.b1791

Katz, R. (2009). Use of revised international health regulations during influenza A (H1N1) epidemic, 2009. Emerging Infectious Diseases, 15, 1165-1170. doi:10.3201/eid1508.090665.

Leung, G. M. et al. (2004). The epidemiology of Severe Respiratory Syndrome in the 2003 Hong Kong epidemic: an analysis of all 1755 patients. Annals of Internal Medicine, 141, 662-673.

Taubenberger, J. K. and Morens, D. M. (2006). 1918 influenza: the mother of all pandemics. Emerging Infectious Diseases, 12, 15-22.

World Health Organization (2000). Preventing Suicide: $A$ Resource for General Physicians (WHO/MNH/MBD/00.1). Geneva: Department of Mental Health, World Health Organization.

World Health Organization (2003). Consensus Document on the Epidemiology of Severe Acute Respiratory Syndrome (SARS). Geneva: Department of Communicable Disease Surveillance and Response, World Health Organization.

World Health Organization (2009a). Pandemic (H1N1) 2009 - Update 58. Laboratory-confirmed cases of pandemic (H1N1) 2009 as officially reported to WHO by States Parties to the International Health Regulations. Available at: http://www.who.int/csr/don/2009_07_06/en/index. html.

World Health Organization (2009b). Depression. Available at: http://www.who.int/mental_health/management/ depression/definition/en/. 